\title{
Metamorfosis de los portales: del hiper-texto a la hiper-red
}

\author{
Por Pablo Lara-Navarra
}

\begin{abstract}
Resumen: Perspectiva de algunas de las tendencias de los portales en la actualidad a partir de las diferentes transformaciones tecnológicas, informativas y organizativas de la sociedad de la información, que ayuda a clarificar la amalgama de ideas que engloban el concepto portal con el fin de aportar o sugerir nuevos escenarios de futuro. Con este fin se realiza una evolución conceptual del término portal, se establece la necesidad de introducir modelos de gestión de contenidos electrónicos en base a una comunicación e información interactiva en forma de red y se realiza una aproximación a las redes sociales como elemento de cambio en el paradigma de los portales.
\end{abstract}

Palabras clave: Portales, Gestión de contenidos, Arquitectura de información, Redes sociales, Internet.

\section{Title: The metamorphosis of portals: from hypertext to hypernet}

Abstract: Perspective on some of the current trends in portal design, based on transformations in technology, information, and organization within the information society. Our purpose is to clarify the amalgamation of ideas that encompass the portal concept, with the goal of contributing or suggesting new scenarios for the future. In an effort to address the need for new models of electronic content management based on a network of interactive information and communication, the article proposes a model similar to social networks as a change agent in the portal paradigm.

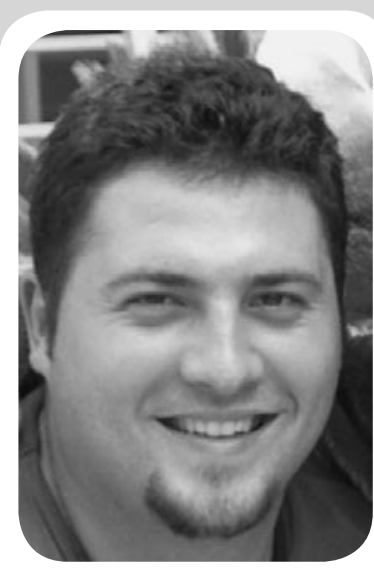

Pablo Lara-Navarra es profesor de los estudios de ciencias de la información y de la comunicación de la Universitat Oberta de Catalunya y miembro del grupo de investigación Ituns-IN3.

Keywords: Portals, Content management, Information architecture, Social networks, Internet.

Lara-Navarra, Pablo. "Metamorfosis de los portales: del hiper-texto a la hiper-red”. En: El profesional de la información, 2007, mayo-junio, v. 16, n. 3, pp. 206-212.

DOI: 10.3145/epi.2007.may.04

\section{Introducción}

CONCEPTUALIZAR Y ESTABLECER EL CONJUNTO de los servicios que debe albergar un portal puede resultar sencillo en su significado más ambiguo de "punto de acceso a". Pero en el momento en que se entremezclan aspectos tecnológicos, organizativos y económicos, sumados a la propia idiosincrasia del concepto de portal, emanan dudas razonables sobre cómo establecer de forma clara y precisa el grupo de servicios y productos para la ardua tarea de captación y fidelización de usuarios/clientes.

En consecuencia surgen diferentes interrogantes, entre ellos: ¿cuáles son los servicios mínimos para optar a la denominación de portal?, ¿qué criterios marcan una tendencia horizontal o vertical de un servicio?, ¿qué elementos de diferenciación se pueden establecer sobre el conjunto de portales existentes?, ¿qué componentes y contenidos han de recoger?

Los preguntas que se nos plantean bajo la categorización de portal son muchas y difíciles de contestar en el cambio de estadio en que nos hallamos, caracterizado por el tránsito de una web plana sin interacción a una centrada y controlada por el usuario denominada web 2.0.

\section{Histórico del concepto portal}

En su significado más genérico de "entrada a" puede ser atribuido a los primeros directorios y motores de búsqueda, indizadores manuales o automáticos utilizados de guía para la exploración de internet y sus contenidos; es decir, portales de acceso a la información clasificada en la web.

Esta acepción, entendida como la base del acceso a un conjunto de recursos de información organizados, queda obsoleta en el momento en que internet se convierte en el medio de comunicación e información universal (remarcando el carácter universal). A partir de este punto el significado de portal ha tomado tantas variantes como personas acceden a internet y como servicios se ofertan a su comunidad de usuarios, desvirtuándose de tal forma que es casi imposible realizar 
una definición que recoja de forma inequívoca sus características. Por consiguiente, este significado evoluciona a la misma velocidad que la tecnología y el uso que las personas hacen de ella.

A continuación presentamos algunas definiciones que muestran la heterogeneidad de ideas en su caracterización. Un portal es entendido como:

"La página web que agrega contenidos y funcionalidades, organizados de tal manera que facilitan la navegación y proporcionan al usuario un punto de entrada en la Red con un amplio abanico de opciones" (Pérez de Leza, 2000).

"Punto de entrada, donde el usuario ve concentrados todos los servicios y productos que ofrece, de forma que le permite hacer cuanto necesita sin tener que salir de un website. Es una forma de captar clientes ya que el objetivo empresarial de cualquier portal es conseguir que su página genere lealtad entre los usuarios, en definitiva, maximizar el tiempo que permanece en sus páginas, antes de saltar a otro destino en la Red y asegurarse que vuelve de manera sucesiva" (Sánchez; Saorín, 2001).

Baró y Ontalba (2002) establecen sus características como puerta de entrada o web de referencia, con servicios gratuitos de utilidad y motor de búsqueda, que busca una viabilidad económica con publicidad y comercio electrónico y con contenidos propios o adquiridos. Quedan excluidos los webs que sean puertas de entrada a una intranet y aquellos que recopilan y estructuran información.

Otras definiciones que se pueden encontrar en internet: "sitios de la world wide web que pretenden transformarse en el principal punto de acceso para los usuarios que se conectan a la web, o en sitio de anclaje que utilizan los usuarios durante la navegación" (Whatis.com); la página de inicio que permite el acceso a las distintas secciones de un sitio web que tiene como servicios chats, e-mail, foros, blogs, etc. (Wikipedia.org).

\section{"Se comprueba claramente una heterogeneidad de ideas en cuanto a las características y funciones de un portal"}

Para terminar con esta introducción, se puede ver en la tabla 1 la relación de los de mayor impacto en el año 2002 según un estudio de Gallup (ahora Investiga). Es posible comprobar como han desaparecido o han cambiado de nombre.

\section{E-contenidos:} elementos de éxito en los portales

La presentación de nuevos modelos de contenidos en base a una comunicación y una información interactiva en forma de red, necesita de una redefinición de las metas tradicionales que proporcione aquellos mecanismos necesarios para alcanzar una gestión de los contenidos electrónicos óptima en los portales. Para ello se han de tener en cuenta al menos los tres requisitos siguientes:

\begin{tabular}{|l|c|l|c|}
\hline \multicolumn{3}{|l|}{ Portales más conocidos } \\
\hline \multicolumn{3}{|c|}{$\%$} & \multicolumn{2}{l|}{} \\
\hline Terra & 79,4 & Wanadoo & 52,0 \\
\hline Yahoo & 43,0 & Navegalia & 34,9 \\
\hline Ya.com & 28,4 & eresMas & 25,3 \\
\hline Lycos & 24,7 & & \\
\hline Portales con más accesos & \\
\hline Terra & 59,3 & Yahoo & 32,8 \\
\hline Wanadoo & 19,6 & eresMas & 15,8 \\
\hline Navegalia & 15,5 & & \\
\hline
\end{tabular}

Tabla 1. Estudio Gallup "Portales de internet. Conocimiento y uso". Abril 2002 a. Estructuras de interacción de contenidos

La realidad del e-contenido se configura a partir de la interacción de los siguientes factores:

- La información como proceso constructivo a la largo de la vida.

- La tecnología a partir de la utilidad comunicativa e informacional que crea nuevos espacios de interacción.

- La organización en tanto que configura la finalidad y el contexto de la actividad del portal.

b. Modelos de actividad del contenido

Las formas de establecer los contenidos de un portal se pueden clasificar en tres grupos de actividades (Roca, 2003):

- De generación: capacidades de producirlos.

- De transformación: acciones que a partir de contenidos generan productos o servicios aptos para el consumo.

- De distribución: permiten a un público final el acceso a unos productos o servicios.

Es decir, hay quien ofrece unos productos (distribuidores) que otros han hecho (transformadores) a partir de materias primas que alguien ha obtenido (generadores). 
Por tanto, un elemento clave para el éxito de la gestión de contenidos es establecer claramente un mapa de relación de los modelos de actividades agregadas con las estructuras de interacciones, para delimitar quién crea contenidos, dónde los desarrolla y cuál es el proceso de actividad aplicado.

El mapa de interacción ha de ser planteado bajo tres ámbitos básicos: organizativo, informativo y tecnológico. Cada uno de ellos puede establecer diferentes modelos. No hay uno único para los mismos; dependerá de quién es el usuario y qué tipo de contenidos requiere. Pero además debemos encontrar el equilibrio y la coherencia entre todos ellos, de forma que el resultado sea lo más satisfactorio para la organización.

c. Configuración de contenidos electrónicos

Este apartado hace hincapié en la importancia de determinar una arquitectura de información óptima que permita una portabilidad de los contenidos ante cualquier cambio que se produzca en el portal. Para una óptima gestión debe establecerse una estructura estandarizada (Lara; Duart, 2005). Es recomendable el uso de lenguaje $\mathrm{xml}$ general, $\mathrm{o} \mathrm{xml}$ específicos si las actuaciones son en campos como la educación electrónica (Scorm, IMS) o administración electrónica $(G O V$ $M L, E M L)$.

El contenedor de esta información necesita de una metainformación que describa correctamente la información que alberga y que será de gran utilidad para los procesos de búsqueda y recuperación de información. Una vez determinados los procesos para generar un contenedor en base a un estándar y una capa con metainformación, se desarrolla un espacio que permita parametrizar el workflow del contenido, que tenga en cuenta para qué fue creado, quién hará uso de él y

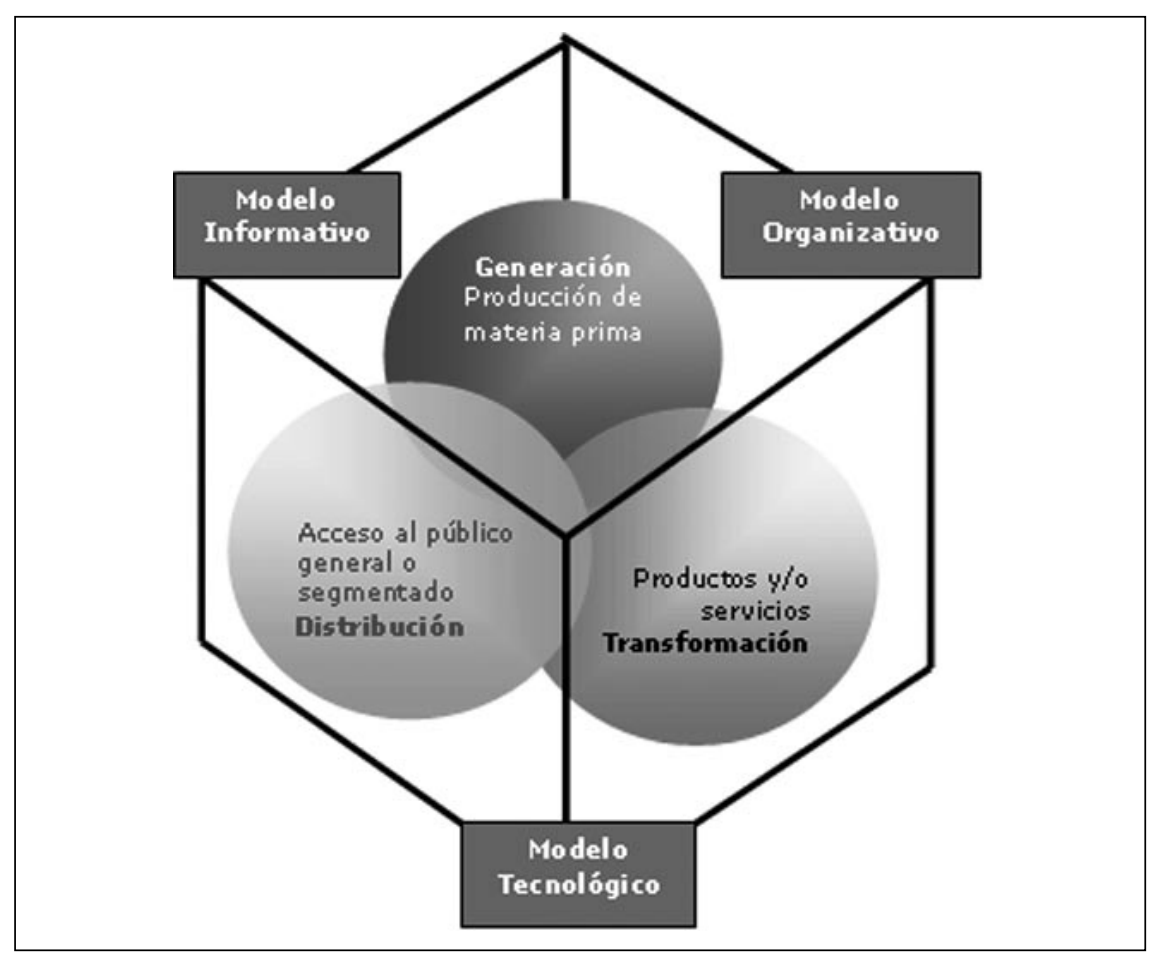

Figura 1. Modelo de actividad en el diseño de contenidos para portales

dónde se realiza la interacción de contenidos.

Todo el conjunto de datos que conforma el contenido ha de ser validado en criterios de usabilidad y accesibilidad y, por último, y no por ello menos importante, en todo caso se han de identificar claramente los derechos de autor y propiedad intelectual que permitirán un uso adecuado y racional de la información.

\section{Evolución de los portales}

Según las características de sus contenidos se pueden agrupar en dos grandes bloques (Franco, 2003):

- Genéricos, también denominados horizontales (horizontal enterprise portal, HEP), e incluso megaportales. Intentan mostrar de manera estructurada todo lo que podría encontrarse en la Red. Aun así, algunos permiten cierto grado de personalización.

-Especializados o verticales (vertical enterprise portal, VEP). Suelen centrarse exhaustivamente en un aspecto dentro de un ámbito geográfico o de una temática (finanzas, actualidad, salud, etc.), o bien adaptan sus contenidos a las necesidades del cliente (tanto en información como en servicios). En este último caso el acceso no es gratuito y se trata de una intranet. Así deben considerarse los portales corporativos que dentro de una intranet de empresa proveen a sus miembros de información interna y externa relacionada con la propia entidad.

Atendiendo a su evolución y como elemento clarificador para ilustrar las diferentes etapas de cambio que han seguido a lo largo del tiempo, se establece la siguiente clasificación:

- Etapa 1: como puertas de acceso.

- Etapa 2: para uso intensivo de información.

- Etapa 3: basados en redes sociales.

4.1. Portales como puertas de acceso

Se encuentran en fase de extinción debido al nivel de experiencia de los usuarios en el uso de los servicios de la sociedad de la 
información, a la evolución de las tecnologías que la sustentan y a la existencia de grandes y exhaustivos buscadores como Google. Se caracterizan por la falta de interacción y sirven como escaparate de los recursos que existen en internet.

Existen pocos casos, ya que la mayoría se han transformado o han desaparecido. Sirvan como ejemplo los citados en la tabla 1 , como $\mathrm{Na}$ vegalia, Eresmas o Wanadoo, que han sido absorbidos por sus matrices, adoptando un nuevo modelo de negocio de servicios hacia el usuario basado en las demandas de las compañías de telecomunicaciones.

\subsection{Portales como concentra- dores de la información}

La interacción directa e ininterrumpida con los contenidos convierte a éstos en artífices importantes y claves en el portal, remarcándose la importancia de su gestión dentro de la organización mediada por tecnología.

Esta realidad dibuja un nuevo contexto de las estructuras de los portales, donde los procesos de creación de información por unidades dejan paso a estructuras "ecológicas" en las que se prioriza el crecimiento transversal. En la figura 2 se observa como los contenidos tienen relación con todas las capas que configuran el portal. Se puede delimitar tres grandes capas básicas: agentes, herramientas y portal.

- Agentes. Empleados y usuarios/clientes.

Tradicionalmente ha recaído la exclusividad de publicación de contenidos en el trabajador de un portal. Este modelo se basaba en la baja interactividad de los usuarios/ clientes con el portal y el sentido unidireccional de la información.

Los cambios hacia una red más dinámica, junto a un rol de uso y un tipo de usuario/cliente con mayor conocimiento en TICs, modifica las estructuras tradicionales de publi- cación. El contenido es más interactivo, ya no es unidireccional sino bidireccional y multidireccional, y tiene funciones de reutilización. Asimismo, el usuario/cliente tiene más predisposición a intervenir en los procesos de generación de contenidos.

- Herramientas de generación de contenidos.

Destacan las relacionadas con los procesos de comunicación y de edición y las de sistemas de gestión. Las primeras, indistintamente de sus funciones y uso, se pueden dividir en asíncronas (correo electrónico, foros, listas de distribución, wikis, blogs, podcasting, etc.) y síncronas (chats, videoconferencias, etc.). Las herramientas de edición ayudan a desarrollar recursos de información en distintos formatos (Word, pdf, html, MP3, etc.) para su posterior distribución.

Las herramientas de sistemas de gestión no tienen que estar supeditadas a un uso exclusivo por parte de los agentes, ya que también sirven para gestionar las acciones que realizan otras herramientas. De este grupo destacan los CMS (content management systems) o sistemas de gestión de contenidos, que se caracterizan por proporcionar soluciones para diseño, maquetación, publica- ción, flujos de trabajo y control de derechos de autor de los contenidos que se generan, y los ECMS (entreprise content management systems) o sistemas de gestión total de contenidos, que combinan las capacidades de gestión con las de almacenamiento y creación de contenidos de un $C M S$.

- Portal.

Es el espacio que ofrece cobertura a todos los sucesos del ciclo de vida de un contenido y que rige su parametrización a partir de los objetivos y metas que se han establecido para su generación. Los contenidos serán el reflejo de la estructura organizativa. Algunos autores afirman que el tipo de contenidos junto a su disposición en una sede web permiten discernir a priori el tipo y estructura de la organización.

Son producidos por todos los elementos internos de la organización pero no todos ellos son internos. Esto se puede observar en la figura 2, en la que la capa de contenidos está presente en todas sus estructuras y a su vez fuera de ella. Esta disposición fuera de la organización sirve para indicar que el portal puede recibir contenidos externos y que éstos son recogidos, organizados y distribuidos para su interacción dentro de su propia organización.

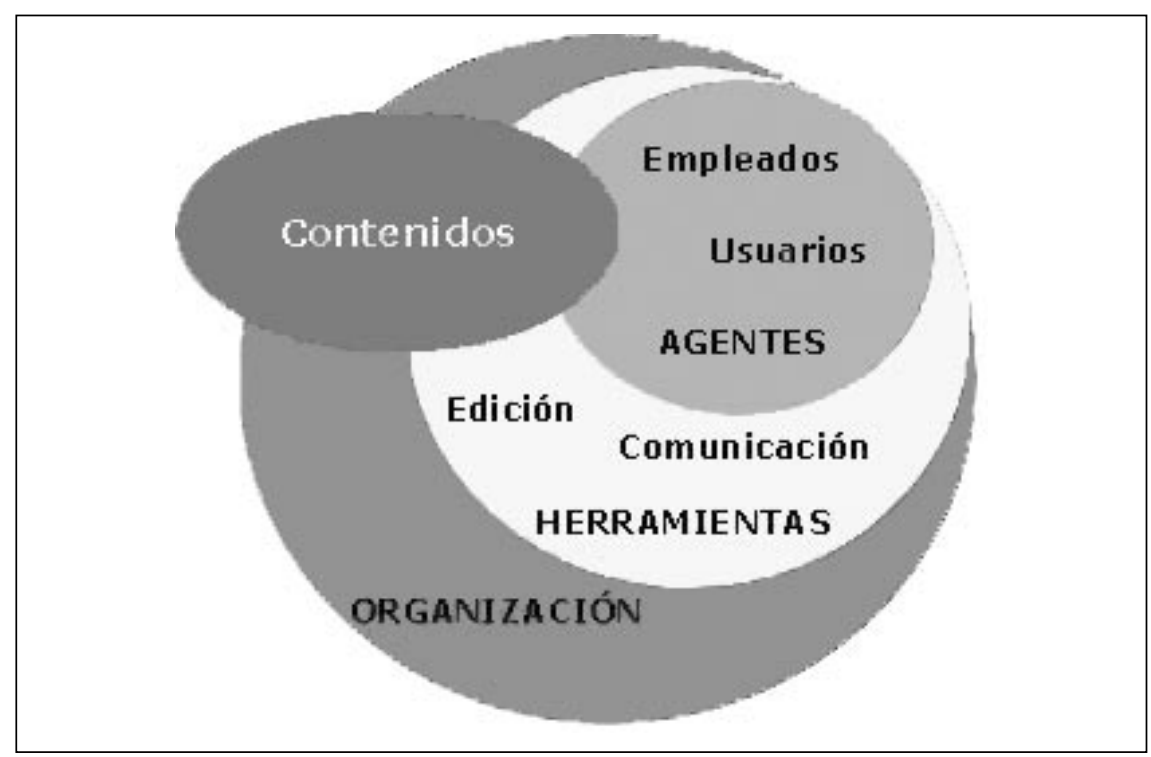

Figura 2. Modelo de interacción de las organizaciones en el uso intensivo de información 
"El portal como concentrador de la información es el más común, siendo su piedra angular las herramientas de gestión de contenidos. Se caracteriza por la interacción con la información y la personalización de espacios"

Algunos ejemplos de portales como concentradores de la información son los siguientes:

Horizontales

http://www.blogger.com*

http://www.youtube.com *

http://www.msn.com *

http://www.yahoo.com *

Verticales

http://www.emagister.com

http://www.infojobs.com

http://www.mailxmail.com

http://www.softonic.com

\subsection{Portales basados en las redes sociales}

Son la gran apuesta hacia donde miran todas las innovaciones que están teniendo lugar en estos momentos en el ámbito de los portales. Hay que señalar sin embargo que es difícil establecer cuál será el resultado final. Aunque se encuentran en un estadio muy evolucionado no es posible acotar categóricamente qué representan o qué tipos de herramientas los integrarán. Sí podemos establecer que los instrumentos colaborativos abiertos a los usuarios como los blogs y los wikis fueron la punta de lanza de este cambio estratégico. Para comprender la evolución de los portales hacia las redes sociales, debemos precisar el concepto y el significado de red.

\subsubsection{El concepto de sociedad red}

La red, a partir de las tecnologías de la información, se define como el conjunto de agentes que se reúnen en torno a un canal de comu-

(*) Hacen uso de herramientas de redes sociales nicación, agrupados por un interés o afinidad común, y que intercambian información para satisfacer sus necesidades sobre el conocimiento que los agrupa. Las tecnologías de la información han permitido que el concepto de inmediatez o sincronía en tiempo real modifique las estructuras de las agrupaciones, a partir de la evolución del concepto red.

Una idea parecida apuntó Castells (1999) al afirmar: "aunque la forma en red de la organización social ha existido en otros tiempos y espacios, el nuevo paradigma de la tecnología de la información proporciona la base material para que su expansión cale en toda la estructura social". Castells no ofrece en su obra una definición clara y concisa de red. La alusión más directa, de la que podemos deducir las características del paradigma de la tecnología de información, aparece cuando escribe que:

"La tercera característica alude a la lógica de interconexión de todo sistema o conjunto de relaciones que utilizan estas nuevas tecnologías de la información. La morfología de red parece estar bien adaptada para una complejidad de interacción creciente y para pautas de desarrollo impredecibles que surgen del poder creativo de esa interacción. Esta configuración topológica, la red, ahora puede materializarse en todo tipo de procesos y organizaciones mediante tecnologías de la información de reciente disposición. Sin ellas, sería demasiado engorroso poner en práctica la lógica de interconexión".

Este acercamiento a la definición lo establece a partir de la propuesta de Kelly (1998), que propugna:
"La única organización capaz de un crecimiento sin prejuicios o un aprendizaje sin guía es la red. Todas las demás topologías limitan lo que puede pasar. Un enjambre de redes es todo bordes y, por ellos, abierta sin que importe por dónde se entre. En efecto, la red es la organización menos estructurada de la que puede decirse que tiene una estructura (...). De hecho una pluralidad de componentes verdaderamente divergentes sólo pueden guardar coherencia en una red. Ninguna otra disposición -cadena, pirámide, árbol, circulo, cubo- puede contener a la diversidad auténtica funcionando como un todo".

\subsubsection{Las redes sociales como} base de la evolución de los portales

Naturalmente, no es el uso de internet el que determina la dinámica de la sociedad red, expresada en las prácticas de vida de las personas. Los comportamientos están influenciados por la posición en la estructura social, por los proyectos y aspiraciones de las personas, su mundo familiar y de amistad, así como por su identidad cultural y personal.

Las estructuras sociales han sido estudiadas desde hace tiempo por la sociología. En cambio, el análisis de redes es un conjunto de métodos sistemáticos para el estudio de las estructuras sociales.

Wellman (1998) distingue el análisis de las redes sociales frente a otro tipo de investigaciones de la siguiente forma:

1. Los actores y sus acciones son vistos de manera interdependiente en lugar de unidades autónomas.

2. Las relaciones entre actores se realizan a través de canales de 
transferencia o de flujos de recursos (materiales, dinero, información, apoyo político, amistad, respeto, etcétera).

3. El enfoque de redes coloca a los individuos en la perspectiva de ofrecer oportunidades y restricciones para sus acciones.

4. La estructura en las redes contiene un patrón de relaciones entre los actores.

Las estructuras de relaciones tienen un poder explicativo más importante que los atributos personales de los miembros que componen el sistema.

- Las normas emergen en función de la localización en la estructura de relaciones existentes.

- Las estructuras sociales determinan el funcionamiento de las relaciones diádicas (duales, de pareja).

-El mundo está formado por redes y no por grupos.

Por otro lado, los prácticos de la aplicación del enfoque de las redes hablan del paradigma de redes desde un punto de vista de los actores y presentan los siguientes elementos clave (Lin; Cook; Burt, 1996):

- Reciprocidad: entendida como buena voluntad de intercambiar información, know-how, conocimientos y bienes.

- Confianza: entendida como disposición de tomar riesgo, depositando la confianza en la fiabilidad de otros.

- Aprendizaje: reconocimiento que el conocimiento desarrolla, debiéndose de aprender las mejores experiencias.

- Asociación: disposición de hacer preferentemente más sólidas las relaciones recíprocas.

- Descentralización: reconocimiento de que la información y toma de decisión centralizados son ineficientes.
Por consiguiente, todos los elementos y características descritos anteriormente son la base de las tecnologías que sustentan el nuevo paradigma de los portales, y se centra en la evolución del concepto de red de documentos (hipertexto) al de red de personas (hiper-red).

\section{"El nuevo paradigma de los portales se centra en la evolución del concepto de red de documentos (hipertexto) al de red de personas (hiper-red)"}

En la figura 3 se representan algunas herramientas tecnológicas que actualmente se encuentran en fase de realización, que cumplen algunos de los criterios anteriormente mencionados y que se incorporan progresivamente a internet en forma de webs sociales. Todo está asentado sobre una premisa: la colaboración. Otro de los rasgos que las caracterizan es el movimiento open o abierto.

Una lista de web sociales se puede consultar en:

http://en.wikipedia.org/wiki/ List_of_social_networking_websites

Algunos ejemplos son los siguientes: http://www.meneame.net

http://www.documenea.com

http://del.icio.us/

http://360.yahoo.com

http://www.eurekster.com

http://www.answers.com

http://www.wikipedia.com

http://www.myspace.com

http://www.orkut.com

\section{Principales conclusiones}

La definición de las posibles formas que puede adoptar un contenido a partir del canal de uso y el formato de creación es considerado un elemento clave en el despliegue de los modelos de portales en la sociedad de la información.

El reto de los portales de uso intensivo de información electrónica se centra en los aspectos clave de comunidad e información; por lo tanto, cualquier acción de gestión de contenidos debe cumplir estas dos exigencias: hacer comunidad y producir información.

La industria de contenidos incorpora una nueva estrategia en los procesos de relación social en general y de las de producción en particular, como se refleja en el auge de participación en estos procesos y la sucesiva institucionalización de la comunicación en forma de redes

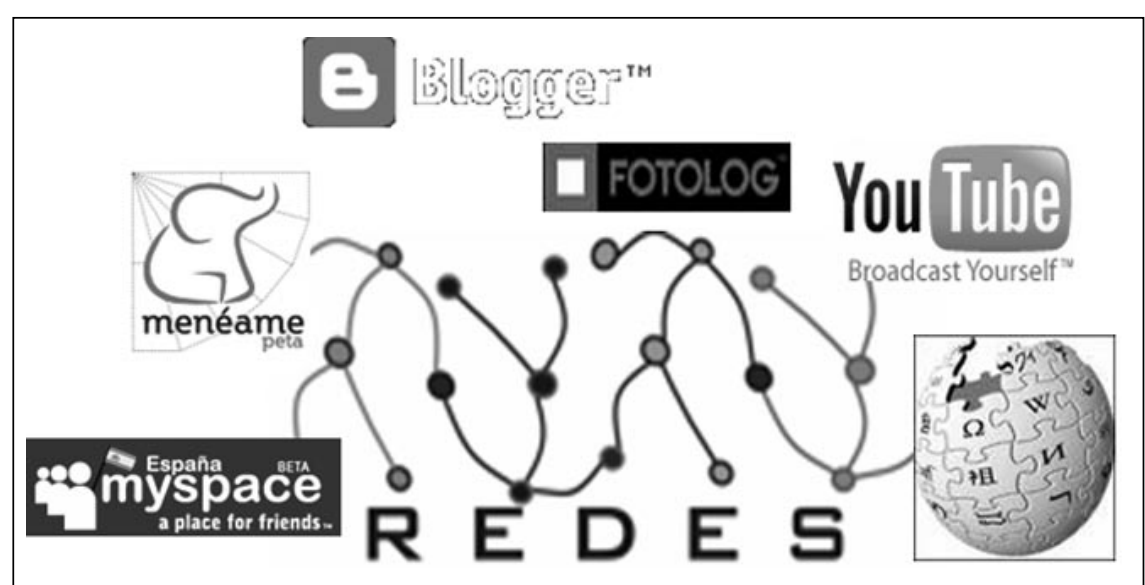

Figura 3: Interacción de las redes con diferentes productos tecnológicos 
"La web 2.0 se caracteriza por un cambio de paradigma donde la información pasa a un segundo nivel y las personas son el centro de la Red"

(Bricall, 2004). Por tanto, se han de concentrar esfuerzos en detectar los nuevos modelos de interacción para la generación y uso de contenidos electrónicos a partir de las teorías de redes sociales.

La evolución indicada podemos definirla como la transformación del concepto de hiper-texto en el de hiper-red, que viene a redibujar el panorama de la web y que obliga a una redefinición de hipervínculo. Donde antes los enlaces o referencias cruzadas automáticas señalaban a otros documentos ahora señalan a otras personas. En consecuencia el hipervínculo se puede redefinir como un elemento de una persona o red que hace referencia a otras personas o redes.

\section{Bibliografía}

Baró, Jaume; Ontalba, José Antonio. Portales españoles: ¿demasiados productos para pocos clientes? Consultado en: 02-03-07.

http://www.uoc.edu/web/esp/art/uoc/0107029/ portales.html

Bricall, J. M. La universidad en el siglo XXI. Barcelona: 2004.

Castells, Manuel. La era de la información: economía, sociedad y cultura, v. 1 (La sociedad red). Madrid: Alianza Editorial, 1999.

Franco, Guillermina. "El portal local: un vehículo estratégico de alfabetización visual para el ciudadano”. En: Razón y palabra, 2005, n. 42.

Kelly, Kevin. Nuevas reglas para la nueva economía. México: Ediciones Granica, 1999. tión de contenidos en el e-learning: acceso y uso
Lara-Navarra, Pablo; Duart, Josep M. "Ges- de objetos de información como recurso estratégico". En: Revista de universidad y sociedad del conocimiento, 2005, v. 2, n. 2.

Lin, Nan; Cook, Karen; Burt, Ronald S. (eds.) Social capital: theory and research. New York, NY: Aldine de Gruyter, 2001.

Molina, José Luis. El análisis de redes sociales. Una introducción. Barcelona: Edicions Bellaterra, 2001.

Pérez-de-Leza, J. "El valor añadido de un portal". En: Ecomm, 2000, v. 13.

Roca, Genís. Modelos de presencia en la red. Consultado en: 05-03-04.

http://www.uoc.edu/web/esp/art/uoc/rocag0502/ rocag0502.html

Sánchez, M.-Vanesa; Saorín, Tomás. "Las comunidades virtuales y los portales como escenarios de gestión documental y difusión de información”. En: Anales de documentación, 2001, v. 4.

Wasserman, Stanley; Faust, Katherine. Social network analysis. Methods and applications. Cambridge: University Press, 1994.

Wellman, Barry. "El análisis estructural: del método y la metáfora a la teoría y la sustancia" En: Política y sociedad, 2000, v. 33.

Pablo Lara-Navarra, Universitat Oberta de Catalunya.

plara@uoc.edu

\section{nature.com} es biología
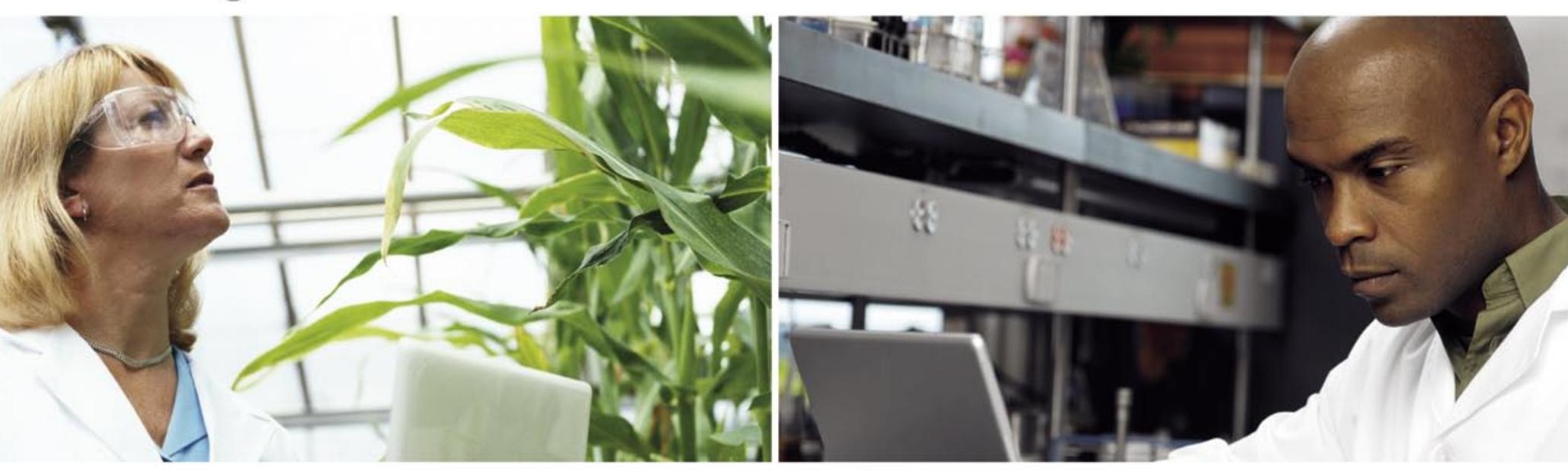

\title{
Está en nature.com
}

\begin{abstract}
Consiga su acceso a nature.com con una Licencia de sitio NPG y elija entre las revistas más valoradas en ciencia y medicina. Su licencia de sitio le garantiza informes de uso, herramientas de promoción, derechos de post-anulación y servicio de atención al cliente.
\end{abstract}

